\title{
Application of stochastic seismic invertion of simulated anneal method in reservoir prediction of Xing'anling Group in Bei14 Block
}

\author{
Shuxin Qiü, a , Chenfan $\mathrm{Liu}^{2, \mathrm{~b}}$ Zhandong $\mathrm{Li}^{3, \mathrm{c}}$ \\ ${ }^{123}$ College of Petroleum Engineering Institute,Northeast Petroleum University,China \\ aquiushuxin@163.com, bbingqer@163.com, ${ }^{\mathrm{c}} 159124755 @ q q . c o m$
}

\section{Keywords: Suderte oilfield;simulated anneal;stochastic invertion;Xing'anling group}

Abstract.Jason stochastic seismic invention method of simulated anneal is applied in developing oilfield blocks,improved reservoir description precision and oil-gas identification.In order to understand the spatial changes of Xing'anling group reservoir in Suderte oilfield Bei14 block, according to seismic,logging and geological information,applies the stochastic seismic invertion method of simulated anneal on Bei14 block, illustrates the distribution and spatial morphology of major reservoirs,occurring as overlapped sandstones vertically,multi-stages in plan,and NE sources.On invention section the ribbon-like or lenticular sandstones are vertical or inclined to source orientation, while on invention section with progradation configuration parallel to source orientation. This method is useful to understand the reservoir character during late development period and allocate boreholes.

Application of invertion in reservoir prediction has been a important method and technology in international Petroleum Industry, the basic purpose of invertion is to use the propagation law of seismic wave in underground medium,through such processes like the data acquisition,processing and interpretation, speculate the space distribution of underground rock and physical parameters, these data can provide an important basis for the exploration and development. The recursive invertion of the first generation is suitable for the early stage of exploration, the vertical resolution of recursive invertion is slightly higher than that of the conventional seismic profile,however,more effective seismic information loss so that not enough to identify and divide effective reservoirs; The recursive invertion and constrained sparse spike of the second generation is under well logging control,mainly refers to the model based,this recursive invertion is under well logging constraint, suitable for the late stage of exploration of relatively less well logging data, the vertical resolution of recursive invertion can reach $10 \mathrm{~m}$.Invertion is limited to seismic wave impedance data or the quasi wave impedance data with special information derived from the acoustic impedance of the reconstructed log,more effective seismic information loss so that can not enough to be directly got other logging curves by invertion,can not carry out fuither reservoir physical property prediction research,either, the accuracy is not enough to solve these problems; The logging interpolation and extrapolation and Depth domain physical propertyinvertion of the third generation is under seismic control,is a seismic constrained log invertion under the condition of enough well logging data,in this invertion core algorithm,genetic algorithm,artificial neural network,chaos algorithm,simulated annealing and other advanced theories are integrated ${ }^{[1-2]}$,the Seismic inversion technique is main based on well log data,more useful information preservation,so that the vertical resolution were improveed an order of magnitude-can reach meter level,can do for study of single sand layer,the recursive invertion of the third generation is a powerful tool for reservoir prediction research of rolling exploration and development in oilfield and adjust development plan stage.

\section{Geological survey of the study area}

Suderte oilfield within the sag in hailaer basin, hinggan mountains within the work area of tectonic framework performance for north-south zoning,east-west blocking's future, the oil layer at the top of the group structure form and the underlying structural form of buried hill of budate group of end face have certain inheritance,structural type is given priority to with fault block,fault nose.Bei 14 work area is located in the middle of Steve souders was a structural high,belongs to the fault block oil 
reservoir,located in between block bei 28 and block bei 16 two faults,the adjacent Wells and 34 shell 12 Wells,purpose layer for hinggan mountains, in the study area are mainly fan-delta sedimentary facies sedimentary system, were divided into three sequence, divided into MSC1,MSC2 and MSC3 from bottom to top.Please keep a second copy of your manuscript in your office. When receiving the paper, we assume that the corresponding authors grant us the copyright to use the paper for the book or journal in question. Should authors use tables or figures from other Publications, they must ask the corresponding publishers to grant them the right to publish this material in their paper.

Because in the sedimentary period,the water turbulent frequentely,the differences between the hydrodynamic conditions in the reservoir group of reservoir physical properties change is bigger.Reservoir thickness change is big,high degree of cementation and the heterogeneity is poor.Among it,X I X II reservoir group sandstone reservoir group is a set of development,poor connectivity of interval,the lithology mainly argillaceous siltstone and gray green mudstone,sandstone and siltstone,lower overall for electrical,with high resistance of single sand layer thickness is thinner; $\mathrm{X}$ III X IV reservoir group overlap on the surface of qian hill reservoir group, curve is shown as the characteristics of high resistivity and low gamma ray,give priority to with coarse sand and gravel lithology,granularity, particle size is cruder than X I X II reservoir group (fig.1).

\section{Feasibility analysis of simulated annealing stochastic inversion}

In Geophysics, the application of simulatedannealing method is often related to the high degree of multiple solutions ${ }^{[5]}$.this technology can be used in many aspects of oil and gas exploration and development,and its impact on the oil industry is becoming more and more profound and extensive.It has three of the most outstanding advantages:one is to realize the fine description and modeling of oil and gas reservoir, quantitative analysis and characterization of heterogeneity in various scales of reservoir;two can be used to quantitatively study the uncertainty of reservoir description, and then study the uncertainty and investment risk of oil and gas exploration and development.Although the underground reservoir is identified,the underground reservoir is unique objectively,but because of the complexity of underground reservoir and the limitation of the known information, the understanding and description of the geological workers to the underground reservoir is uncertain;Three is to integrate a variety of sources of information and data,and unify them in the same quantitative model ${ }^{[4]}$.In the field of geophysical

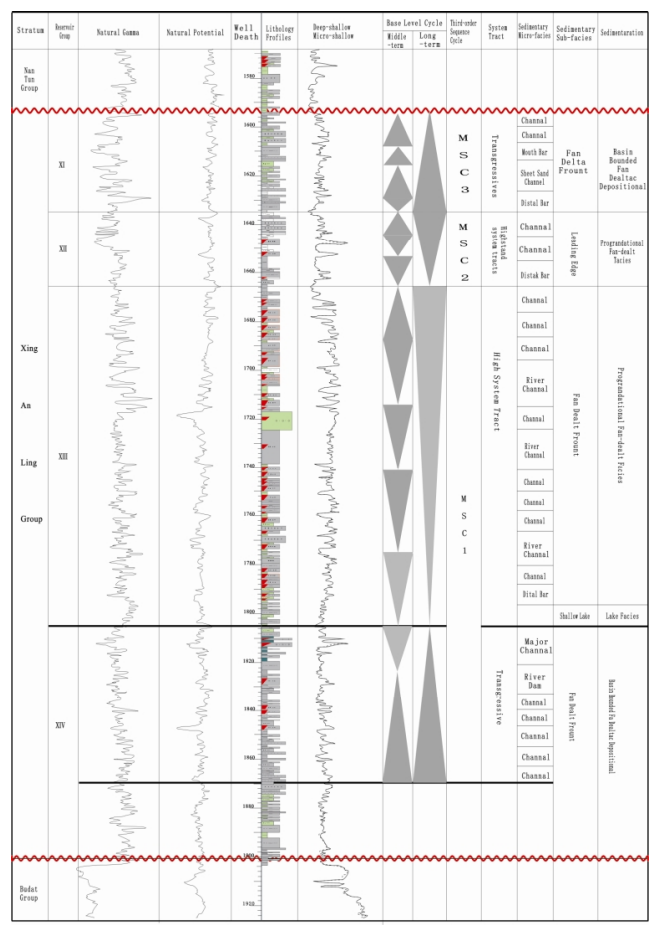

Fig. 1 De 120-175 single well comprehensive map reservoir inversion, the invertion based on simulated annealing algorithm has been paid more attention to because of its flexibility, adaptability and strong synthesis.

The simulated annealing method can be used to simulate the continuous or discrete attribute-body, the simulated annealing method has the advantages that the space structure of the reservoir reflected by known data can be well maintained,it is suitable for reservoir in fluvial, Delta, turbidite environment formation reservoir description, to provide a set of equal probability reservoir models to meet the law of geological statistics,so as to overcome the uncertainty of reservoir simulation.

In the development stage of Suderte oilfield,the study of the reservoir heterogeneity and the expanded boundary project of well area are the oilfield always eager to solve the problem. Based on the above situation, use traditional seismic analysis techniques to predict the thickness of sandstone is difficult to achieve satisfactory result.By using well logging curve and statistical analysis of 
lithology,we can get,the relativity of the thickness of the sandstone and the deep lateral resistivity curve is relatively large(fig.2). Resistivity curve is the most sensitive to lithology response in this area,therefore, in the curve selection, the resistivity curve was chosen as the main,at the same time,using the well seismic invertion to describe the connection of the sand body in the well.therefore, amplitude invertion and simulated annealing inversion are the best invertion method, it can not only enhance the lateral continuity of earthquake,but also can improve the vertical resolution.

\section{Basic principle of simulated annealing}

The concept of simulated annealing is derived from statistical physics, is a physical process that the simulation of the solid state is gradually slow cooling after cooling and finally reaching the crystalline state. When solid in the molten state, it contain the maximum internal energy,but when solid condensed into regular crystal, It contains the minimum internal energy.For a molten metal, very molecule of it is likely to be in a state of itself,and the total energy of the metal is determined by the sum of these molecular states. When the thermal equilibrium is reached, the state of each molecule satisfies the Gibbs probability distribution. While the system is slow to cool,each molecule obtains its own state by Gibbs probability distribution. With the decrease of temperature, the substance finally reaches the crystalline state, at this time, the total energy of the substance is the smallest.here, the state of each molecule is the state of the substance to achieve the minimum energy.
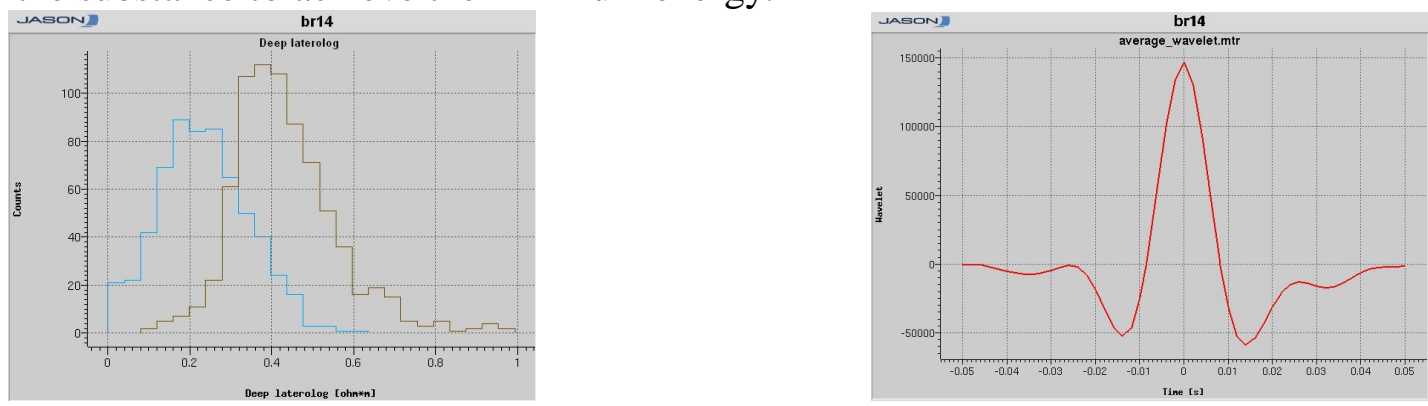

Fig. 2 Lateral resistivity curve and lithology fitting histogram

The process of simulated annealing and Tthe process of solving the invertion problem have similarities that they are both form the error energy of the larger state, and finally to achieve the lowest error energy state.Each molecule in the simulated annealing is equal to the parameters to be retrieved,all the molecules can be selected as the model space equivalent to the invertion,the total energy of the substance determined by all molecules is equivalent to the energy function in the invertion,the state of the molecules in the crystalline state is equivalent to the final results of the invertion,so it is the global optimal value of the minimum energy.Set a model parameter Xi desirable $\mathrm{N}$ possible value $\mathrm{Vj}(\mathrm{j}=1,2 \ldots$ any of the $\mathrm{N})$, in order to obtain a new value of $\mathrm{Xi}$, a random number will be extracted ba the following probability distribution function.

$$
P\left(x_{i}=v_{j}\right)=\frac{\exp \left[-E\left(v_{j}\right) / T\right]}{\sum_{i=1}^{N} \exp \left[-E\left(v_{i}\right) / T\right]}
$$

$\mathrm{E}(\mathrm{Vj})$ is the system energy at $\mathrm{xi}=\mathrm{vj}, \mathrm{T}$ is temperature,this iterative change may be choose anyone of the values $\mathrm{N}$,but $\mathrm{Vj}$ making the $\mathrm{E}$ small is more likely to be selected.This is different from the linear invertion,so it can jump out of local extremum,this is a completely nonlinear invertion method.In this study, $\mathrm{N}=10$, the minimum tolerance value is 0.01 , the maximum tolerance is 0.2 , the model $\mathrm{Xi}$ generated by sequential Gauss simulation is used as the initial model.

\section{Application effect and comprehensive description}

By optimizing the inversion parameters of the region and comprehensive analyzing of a variety of information,the multiple solutions of seismic inversion are reduced to a certain extent ${ }^{[1,2]}$. The inversion result and the well logging data are better corresponded,the spatial connection of sand body reflects the change of reservoir space, in vertical,the reservoir spaces are multi sets of sandstone, while on space,the 
characteristics of the reservoir space are multi period,on the horizontal, the sand body of the oil layer is characterized in front of the west.The comparison of lithology data of well and invertion profile can be seen that the inversion results are in good agreement with the well.By means of the statistics of the thickness of sandstone and the predicted thickness of the drilling in this block, the difference between the thickness of the sand body after prediction and the actual drilling thickness is about $3 \mathrm{~m}$, and the absolute error is less than $5 \%$,so that it can provide a reliable evidence for the geological analysis ${ }^{[9]}$.

his time, the invertion and the the formation of sand bodys correspond well,overall,the higher the resistance rate is, the better the physical properties of the sand bodies are ${ }^{[10,11]}$. The reservoir development of MSC1 in the X water inlet area is relatively good,this sandstone reservoir is under fan-delta and shallow lagoon facies controlled,the lithology is mainly dominated by the gray green mudstone, fine sandstone and muddy sandstone, is a set of forward sedimentary cycle,thickness of sandstone is between $2.4-59.4 \mathrm{~m}$,efective thickness is between $0-16.1 \mathrm{~m}$; the reservoir development of X III oil reservoir in the high water level system is the best,tthis target stratum belongs to the underwater stream channel of fan delta front facies, interchannel and far sand dam microfacies, the lithology of this reverse sedimentary cycle give first place to sandy gravel, coarse sand and fine sandstone, siltstone and fine sand,is the main reservoir area,five sets of sand bodies can be identified in the longitudinal direction, thickness of sandstone is between 10.2-134.1m,efective thickness is between 5.1-90.2m;The reservoir of X I oil layer and X II oil layer is relatively less developed in the well area,thickness of sandstone is between $0-28.2 \mathrm{~m}$,efective thickness is between $0-2.0 \mathrm{~m}$.

Combined with geologic analys, the sediment source direction of study area is the northeast to the sedimentary system, in the inversion section,the shape of the sand body with a strip shape or lens is shown as the source of vertical or oblique direction ${ }^{[12]}$, and in the inversion profile, it shows the phenomenon of the former product structure and the direction of the source of the material..,the thickness of the sand body in the plane thin or pinch from the northeast to the southwest,the sand body of X I and X II oil reservoir set influenced by the offsets of the boundary,and be basically pinched-out in 58 well, X III and X IV oil group to the 62 wells 64 wells are pinched-out about 2-3well away from the northeast,the lateral continuity of sand body not good is the basic reason of The reservoir is not developed and the development of this area is not ideal.but the sand body of X III and X IV oil reservoir set in the North West of the Bei14 fault block,its development is characterized by small flakes,and are gradually pinched-out to the frount,and the typical fault lithologic reservoir is constructed,the remaining oil is distributed in the edge of the reservoir, and this type of reservoir is the key goal of the next development.

\section{References}

[1] Fwngchang Yao, Lideng Gan: Petroleum exploration and development Vol. 34 (2000), p. 53

(in Chinese)

[2] Yonggang Zhang: Geophysical prospecting for petroleum Vol. 12 (2002), p. 385(in Chinese)

[3] Yonggui Zhang: Journal of southwest petroleum institute Vol. 8 (1997), p. 1(in Chinese)

[4] Jianyu Zhou: Petroleum ecology and recovery efficiency Vol. 6 (2005), p. 45(in Chinese)

[5] Cheng Yin: Geophysical prospecting for petroleum Vol. 3 (1998), p. 63(in Chinese)

[6] Dequan Kang: Resources\&Industries Vol. 11 (2009), p. 82(in Chinese)

[7] Xingyou Gao, Ying Li: Resources\&Industries Vol. 8 (2006), p. 98(in Chinese)

[8] Qiaoxia Liu, Renfei Tian, Zhouchan Zhong: Journal of Yangtze University(Nat Sci Edit) Vol. 6 (2009), p. 186(in Chinese)

[9] Dequan Kang: Resources\&Industries Vol. 11 (2009), p. 140(in Chinese) 